\title{
Review \\ Plant-Derived Protectants in Combating Soil-Borne Fungal Infections in Tomato and Chilli
}

\author{
Himanshu Arora ${ }^{1} \mathbb{D}$, Abhishek Sharma ${ }^{2, * \mathbb{D}}$, Peter Poczai ${ }^{3, * \mathbb{D}}$, Satyawati Sharma ${ }^{1} \mathbb{D}$, Farah Farhanah Haron ${ }^{4}$, \\ Abdul Gafur ${ }^{5}$ and R. Z. Sayyed ${ }^{6}$ (D)
}

1 Centre for Rural Development and Technology, Indian Institute of Technology, New Delhi 110016, India; himanshuarora592@gmail.com (H.A.); satyawatis@hotmail.com (S.S.)

2 Amity Food and Agriculture Foundation, Amity University, Noida 201313, India

3 Botany Unit, Finnish Museum of Natural History, University of Helsinki, P.O. Box 7, FI-00014 Helsinki, Finland

4 Biological Control Programme, Agrobiodiversity and Environment Research Centre, Malaysian Agricultural Research and Development Institute, Serdang 43400, Malaysia; farahfarhanah@mardi.gov.my

5 Sinarmas Forestry Corporate Research and Development, Perawang 28772, Indonesia; gafur@uwalumni.com

6 Department of Microbiology, PSGVP Mandal's Arts, Science, and Commerce College, Shahada 425409, India; sayyedrz@gmail.com

* Correspondence: asharma5@amity.edu (A.S.); peter.poczai@helsinki.fi (P.P.)

check for updates

Citation: Arora, H.; Sharma, A.; Poczai, P.; Sharma, S.; Haron, F.F.; Gafur, A.; Sayyed, R.Z. Plant-Derived Protectants in Combating Soil-Borne Fungal Infections in Tomato and Chilli. J. Fungi 2022, 8, 213. https:/ / doi.org/10.3390/jof8020213

Academic Editor: Gary A. Strobel

Received: 19 January 2022

Accepted: 19 February 2022

Published: 21 February 2022

Publisher's Note: MDPI stays neutral with regard to jurisdictional claims in published maps and institutional affiliations.

Copyright: (C) 2022 by the authors. Licensee MDPI, Basel, Switzerland. This article is an open access article distributed under the terms and conditions of the Creative Commons Attribution (CC BY) license (https:// creativecommons.org/licenses/by/ $4.0 /)$.

\begin{abstract}
Fungal infections transmitted through the soil continue to pose a threat to a variety of horticultural and agricultural products, including tomato and chilli. The indiscriminate use of synthetic pesticides has resulted in a slew of unintended consequences for the surrounding ecosystem. To achieve sustainable productivity, experts have turned their attention to natural alternatives. Due to their biodegradability, varied mode of action, and minimal toxicity to non-target organisms, plantderived protectants (PDPs) are being hailed as a superior replacement for plant pesticides. This review outlines PDPs' critical functions (including formulations) in regulating soil-borne fungal diseases, keeping tomato and chilli pathogens in the spotlight. An in-depth examination of the impact of PDPs on pathogen activity will be a priority. Additionally, this review emphasises the advantages of the in silico approach over conventional approaches for screening plants' secondary metabolites with target-specific fungicidal activity. Despite the recent advances in our understanding of the fungicidal capabilities of various PDPs, it is taking much longer for that information to be applied to commercially available pesticides. The restrictions to solving this issue can be lifted by breakthroughs in formulation technology, governmental support, and a willingness to pursue green alternatives among farmers and industries.
\end{abstract}

Keywords: plant secondary metabolites; botanical pesticides; plant diseases; essential oil; soil amendments; in silico

\section{Introduction}

Synthetic pesticides have long been used to effectively manage plant diseases. However, their prolonged and persistent use has resulted in many detrimental and unprecedented effects on the surrounding environment. Pesticide misuse has resulted in many phytopathogens becoming resistant [1]. Pesticides' bioaccumulation and toxicity to non-target organisms have also had negative environmental repercussions [2]. Synthetic pesticides cause nearly two lakhs of deaths from poisoning each year, and $99 \%$ of them occur in developing countries [3]. Although synthetic pesticides are sometimes more convenient, biopesticides derived from natural resources are a superior option. This last decade has seen the various botanicals becoming increasingly prominent in the field of plant protection.

Plant-derived (botanical) protectants (PDPs) provide a competitive advantage over synthetic pesticides because they are safer and cheaper. We employ the term "plant-derived protectants" throughout this article to refer to purified active metabolites, crude plant 
extracts, essential oils, and total phytobiomass. They have multiple modes of action, are biodegradable, and reduced non-target toxicity [4-6]. Regardless of the form of PDPs used, their pesticidal activity is mainly due to bioactive secondary metabolites. Secondary metabolite synthesis is part of the plant's defense strategy. These metabolites might play a crucial role in the health of the plant, but they predominantly act as plant defense agents [7]. In accordance with the biosynthetic principle, plant secondary metabolites can be divided into three main classifications, which are terpenes, phenolics, and nitrogen-containing chemicals (Figure 1) [8]. Terpenes are the most abundant and significant class of secondary metabolites. Monoterpenes are found in abundance in essential oils, which account for about $80 \%$ of the total [9]. Phenols are a class of chemical compounds with a wide range of sizes and miscibility in water and organic solvents. The single-substituted phenolic rings are typically found in simple phenolics, while the complex compounds have phenolic rings connected to several functional groups. Nitrogen-containing secondary metabolites are usually biosynthesised amino acid derivatives.

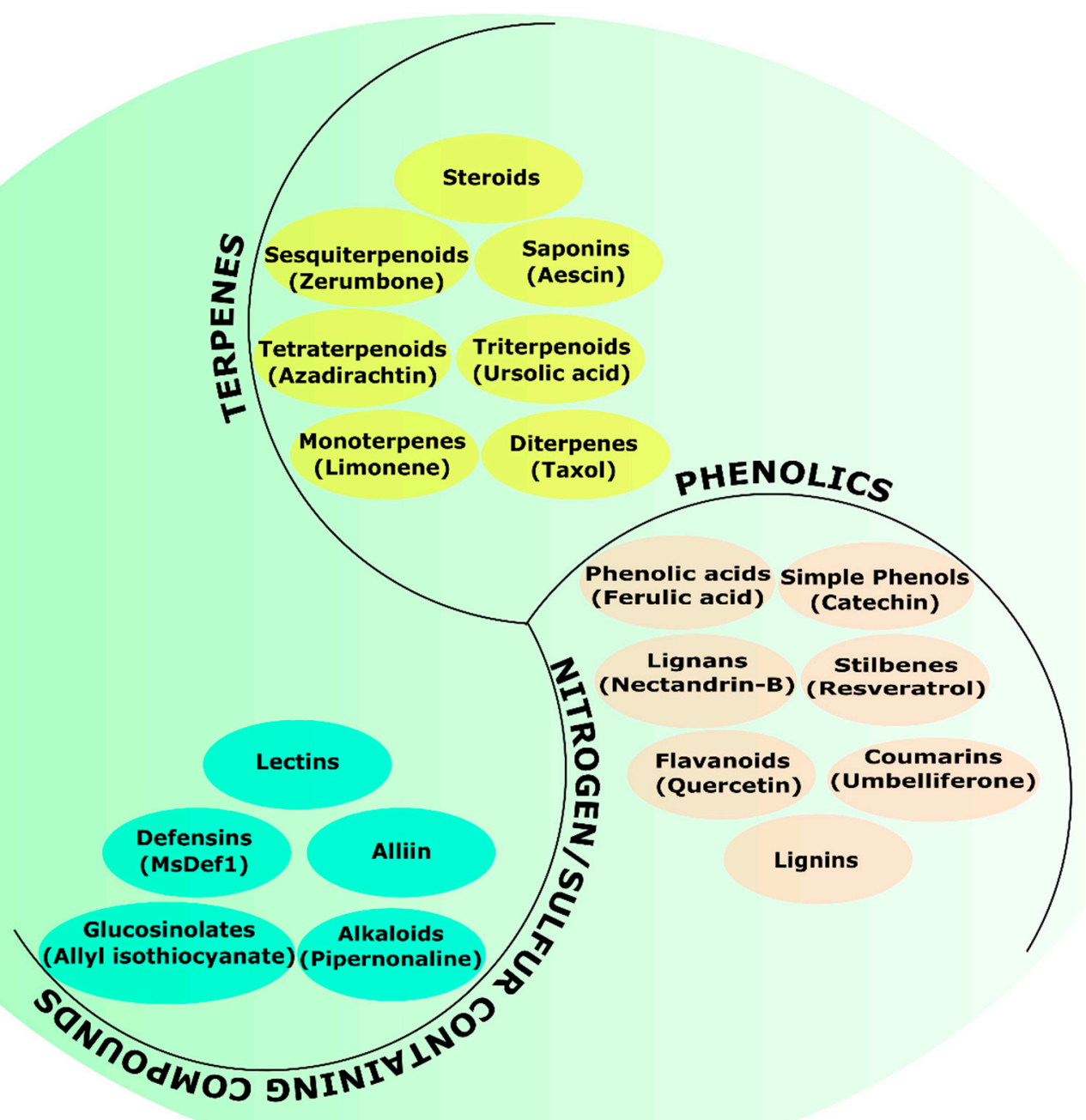

Figure 1. Types of secondary metabolites in plants.

According to the Food and Agriculture Organization (FAO, 2019), worldwide production of tomatoes was almost 180 million tonnes, with dry chiles and peppers at over 4 million tonnes. Unfortunately, tomato and chilli fungal infections have held down market demand for these products. These infections are a hidden, but frequent, roadblock in making tomato and chilli crops profitable. Diseases caused by soil-borne fungal phytopathogens 
such as Fusarium spp., Rhizoctonia spp., Phytophthora spp., Pythium spp., Sclerotinia spp., and Verticillium spp. have resulted in substantial yield losses in these two crops [10-14]. In tomato production, vascular wilt induced by Fusarium spp. involves infection of plants roots, and once this is complete, the vascular system attacks accelerate, leading plants to wilt and lose $10-80 \%$ of their yield [14]. Phytophthora capsici, a fungus that causes root and crown rot in peppers, has led to disease losses of up to $40 \%$ [13]. As with damping-off, Pythium species induce premature death of chilli seedlings in nurseries and greenhouses [10].

PDPs could be a promising tool to regulate soil-borne fungal diseases in Solanum lycopersicum (Tomato) and Capsicum annuum (chilli). Hence, this analysis focuses on every facet of scientific development, starting with screening PDPs, to assessing their application possibilities regarding ways of functioning against fungal phytopathogens of tomatoes and chilli. Additionally, this assessment throws light on enigmas in the process of successfully commercialising scientific endeavors.

\section{Management of Soil-Borne Diseases in Tomato and Chilli}

2.1. Using Crude Plant Extracts, Essential Oils, and Purified Secondary Metabolites (Lab-to-Land Approach)

Applying botanicals or their metabolites straight to the field is likely to yield a variable result [15]. To circumvent this constraint, researchers are designing potent formulations (a combination of one or more active substances and inert components) capable of efficiently managing a pest over an extended period of time in the field [4]. Such "lab to land" strategies boost farmers' abilities to combat crop disease and add economic and societal value to research. Contrary to this fact, we continue to see research using unformulated PDPs, even for field evaluations of disease control.

More than any other soil-borne pathogen in tomatoes, Fusarium management has been studied extensively (Table 1). Purified pomegranate peel water extract (Pae) inhibited mycelial development of $F$. oxysporum f. sp. lycopersici by $83 \%$ at a concentration of $0.5 \%$ $(w / v)$ with higher concentrations of phenolic acid-like punicalagins and ellagic acid [16]. When employed in soil treatment, pure Pae was likewise effective at reducing disease incidences in treated tomato plants to about $58 \%$ in treatment when compared to $100 \%$ in the untreated control. Among others, extracts of Allium tuncelianum, A. sativum, Azadirachta indica, Zingiber officinale, Pistacia lentiscus, Moringa oleifera, Stevia rebaudiana, Theobroma cacao, Juglans macrocarpa, and J. mollis successfully inhibited the disease and symptom development in tomato plants against various Fusarium species [13,17-21]. Tomato plants cultivated in a non-circulating hydroponic system were treated with Thymbra capitata essential oil at $1.473 \mu \mathrm{L} / \mathrm{L}$ concentration, resulting in a $30.76 \%$ reduction in disease severity caused by F. oxysporum f. sp. radicis-lycopersi [22]. A study evaluating various essential oils for antifungal activity against F. oxysporum f. sp. lycopersici discovered that Syzygium aromaticum oil was the most effective. The oil was later utilised as a $5 \%$ aqueous emulsion where it exhibited an $86.5 \%$ decrease in Fusarium wilt in tomato during a pot trial [23]. A subsequent investigation explored a 5\% $(w / w)$ nanoemulsion formulation that used the previously mentioned Syzygium aromaticum and Cymbopogon citratus essential oils for synergistic potential. The formulation at $4000 \mathrm{mg} / \mathrm{L}$ provided $67.51 \%$ wilt disease control [24]. The finding demonstrates that synergy is a novel notion that should be investigated more regularly for the purpose of ensuring sustained plant protection. At a $1.5 \%$ ethanol extract concentration, Ozkaya and Ergun [13] found Allium tuncelianum to be efficient against Pythium deliense, Rhizoctonia solani, Sclerotinia sclerotiorum, and Sclerotium rolfsii. The investigation using Euphorbia latex derivatives to control Verticillium dahliae in tomatoes found that the seed treatment reduced symptoms significantly [25,26]. Mekam et al. [27] observed that spraying Euphorbia hirta leaf ethanolic extract at $2.50 \mathrm{mg} / \mathrm{mL}$ offered protection against soil-borne Rhizoctonia solani. Table 1 summarises botanicals employed to control soil-borne fungal diseases of tomatoes. 
Table 1. PDPs reported to control soil-borne diseases in the tomato.

\begin{tabular}{|c|c|c|c|c|c|c|c|}
\hline Pathogen & Source Plant & Plant Part & Solvent & $\begin{array}{l}\text { Major Bioactive } \\
\text { Compounds }\end{array}$ & In-Vitro Control & $\begin{array}{c}\text { In Vivo Disease } \\
\text { Control }\end{array}$ & Reference \\
\hline \multirow{2}{*}{ V. dahliae } & $\begin{array}{l}\text { Euphorbia } \\
\text { officinarum }\end{array}$ & Latex & Not available & $\begin{array}{c}\text { Oxidation } \\
\text { derivatives of } \\
\text { 31-norlanostenol }\end{array}$ & $\begin{array}{l}\text { No inhibition at } \\
10 \mu \mathrm{g} / \mathrm{mL} \\
\text { compound } \\
\text { concentration }\end{array}$ & $\begin{array}{l}\text { Seed treatment in } \\
5 \mathrm{~mL} \text { of } 10 \mu \mathrm{g} / \mathrm{mL} \\
\text { compound } \\
\text { concentration of } \\
\text { derivatives reduced } \\
\text { the disease } \\
\text { symptoms }\end{array}$ & \multirow{2}{*}[25]{} \\
\hline & $\begin{array}{l}\text { Euphorbia } \\
\text { resisnifera }\end{array}$ & Latex & Not available & $\begin{array}{c}\text { Oxidation } \\
\text { derivatives of } \\
\alpha \text {-euphorbol }\end{array}$ & $\begin{array}{l}\text { Insignificant } \\
\text { inhibition at } \\
10 \mu \mathrm{g} / \mathrm{mL} \\
\text { compound } \\
\text { concentration }\end{array}$ & $\begin{array}{l}\text { Seed treatment in } \\
5 \mathrm{~mL} \text { of } 10 \mu \mathrm{g} / \mathrm{mL} \\
\text { compound } \\
\text { concentration of } \\
\text { derivatives reduced } \\
\text { the disease } \\
\text { symptoms }\end{array}$ & \\
\hline V. dahliae & $\begin{array}{l}\text { Euphorbia } \\
\text { officinarum }\end{array}$ & Latex & Not available & $\begin{array}{c}\text { Oxidation } \\
\text { derivatives of } \\
\text { lupeol acetate } \\
\text { and } \\
\text { 31-norlanostenol }\end{array}$ & $\begin{array}{l}\text { 56-60\% reduction } \\
\text { in conidia } \\
\text { formation at } \\
100 \mu \mathrm{g} / \mathrm{mL} \\
\text { compound } \\
\text { concentration }\end{array}$ & $\begin{array}{l}\text { Spraying of seedling } \\
\text { with } 10 \mu \mathrm{g} / \mathrm{mL} \\
\text { compound } \\
\text { concentration of } \\
\text { derivatives reduced } \\
\text { the disease } \\
\text { symptoms }\end{array}$ & [26] \\
\hline \multicolumn{8}{|l|}{ Essential oil } \\
\hline \multirow{2}{*}{$\begin{array}{l}\text { F. oxysporum f. } \\
\text { sp. lycopersici }\end{array}$} & $\begin{array}{l}\text { Thymus } \\
\text { vulgaris }\end{array}$ & Not available & - & $\begin{array}{l}\text { Thymol, } \\
\alpha \text {-pinene }\end{array}$ & $\begin{array}{l}\mathrm{MIC}_{50}{ }^{\mathrm{A}}= \\
152 \mu \mathrm{g} / \mathrm{mL}\end{array}$ & $\begin{array}{l}\text { Soil treatment with } \\
300 \mu \mathrm{g} / \mathrm{mL} \text { oil } \\
\text { concentration } \\
\text { resulted in } 32.2 \% \\
\text { efficacy in disease } \\
\text { severity reduction }\end{array}$ & \multirow{2}{*}{ [28] } \\
\hline & $\begin{array}{c}\text { Eugenia } \\
\text { caryophyllata }\end{array}$ & Not available & - & Eugenol & $\begin{array}{c}\mathrm{MIC}_{50}= \\
172 \mu \mathrm{g} / \mathrm{mL}\end{array}$ & $\begin{array}{l}\text { Soil treatment with } \\
300 \mu \mathrm{g} / \mathrm{mL} \text { oil } \\
\text { concentration } \\
\text { resulted in } 42.4 \% \\
\text { efficacy in disease } \\
\text { severity reduction }\end{array}$ & \\
\hline $\begin{array}{l}\text { F. oxysporum f. } \\
\text { sp. lycopersici }\end{array}$ & $\begin{array}{l}\text { Syzygium } \\
\text { aromaticum }\end{array}$ & Not available & - & $\begin{array}{c}\text { Eugenol, } \\
\text { E-caryophyllene, } \\
\alpha \text {-humulene, } \\
\text { caryophyllene } \\
\text { oxide, }\end{array}$ & $\begin{array}{l}\mathrm{IC}_{50}{ }^{\mathrm{B}}=18.22 \mathrm{ppm} ; \\
\mathrm{MIC}^{\mathrm{C}}=31.25 \text { ppm; } \\
\mathrm{MFC}^{\mathrm{D}}=125 \text { ppm }\end{array}$ & $\begin{array}{l}86.5 \% \text { reduction in } \\
\text { disease incidence } \\
\text { when } 5 \mathrm{~mL} \text { of } 5 \% \\
\text { aqueous emulsion of } \\
\text { essential oil used for } \\
\quad 150 \mathrm{~cm}^{3} \text { soil }\end{array}$ & [23] \\
\hline $\begin{array}{l}\text { F. oxysporum f. } \\
\text { sp. lycopersici }\end{array}$ & $\begin{array}{c}\text { Syzygium } \\
\text { aromaticum }+ \\
\text { Cymbopogon } \\
\text { citratus }(1: 1)\end{array}$ & Not available & - & $\begin{array}{c}\text { Eugenol, } \\
\text { E-caryophyllene } \\
\text { and Geranial, } \\
\text { Neral }\end{array}$ & $\begin{array}{l}\text { For the } 5 \%(w / w) \\
\text { nanoemulsion } \\
\text { prepared } \\
\mathrm{MIC}=4000 \mathrm{mg} / \mathrm{L} ; \\
\mathrm{MFC}=5000 \mathrm{mg} / \mathrm{L}\end{array}$ & $\begin{array}{c}67.51 \% \text { disease } \\
\text { control when } 5 \mathrm{~mL} \text { of } \\
4000 \mathrm{mg} / \mathrm{L} \\
\text { concentration of } 5 \% \\
(w / w) \text { nanoemulsion } \\
\text { used for } 150 \mathrm{~cm}^{3} \text { soil } \\
\text { treatment }\end{array}$ & [24] \\
\hline F. solani & $\begin{array}{l}\text { Oreganum } \\
\text { vulgare }\end{array}$ & Not available & - & Not available & $\begin{array}{c}\text { For the } \\
\text { emulsifiable } \\
\text { concentrate } \\
\text { prepared, } 100 \% \\
\text { mycelial inhibition } \\
\text { at } 4000 \text { ppm } \\
\text { concentration }\end{array}$ & $\begin{array}{l}\text { Seed treatement with } \\
4000 \text { ppm } \\
\text { concentration of } \\
\text { emulsifiable } \\
\text { concentrate for } 8 \mathrm{~h} \\
\text { resulted in } 50 \% \\
\text { reduction in } \\
\text { pre-emergence } \\
\text { damping-off }\end{array}$ & [29] \\
\hline
\end{tabular}


Table 1. Cont.

\begin{tabular}{|c|c|c|c|c|c|c|c|}
\hline Pathogen & Source Plant & Plant Part & Solvent & $\begin{array}{l}\text { Major Bioactive } \\
\text { Compounds }\end{array}$ & In-Vitro Control & $\begin{array}{c}\text { In Vivo Disease } \\
\text { Control }\end{array}$ & Reference \\
\hline $\begin{array}{l}\text { F. oxysporum f. } \\
\text { sp. radicis } \\
\text { lycopersici }\end{array}$ & $\begin{array}{l}\text { Foeniculum } \\
\text { vulgare }\end{array}$ & Seeds & - & $\begin{array}{l}\text { Trans-anethole, } \\
\text { L-fenchone, } \\
\text { Estragole, } \\
\text { Limonene }\end{array}$ & $\begin{array}{l}83 \% \text { reduction in } \\
\text { mycelial growth at } \\
500 \mu \mathrm{L} / \mathrm{mL} \text { oil } \\
\text { concentration }\end{array}$ & $\begin{array}{l}40-60 \% \text { reduction in } \\
\text { disease severity } \\
\text { when the soil was } \\
\text { drenched with } 50 \mathrm{~mL} \\
\text { of } 500 \mu \mathrm{L} / \mathrm{mL} \text { oil } \\
\text { concentration }\end{array}$ & [30] \\
\hline \multicolumn{8}{|l|}{ Plant extract } \\
\hline $\begin{array}{l}\text { F. oxysporum } \mathrm{f} \text {. } \\
\text { sp. lycopersici; } \\
\text { P. deliense; } \\
\text { R. solani; } \\
\text { S. sclerotiorum; } \\
\text { S. rolfsii }\end{array}$ & $\begin{array}{c}\text { Allium } \\
\text { tuncelianum }\end{array}$ & Not available & $96 \%$ ethanol & Not available & Not available & $\begin{array}{l}\text { Soil treatment with } \\
10 \mathrm{~mL} \text { of } 1.5 \% \text { extract } \\
\text { significantly reduced } \\
\text { the disease severity } \\
\text { against all pathogens }\end{array}$ & [13] \\
\hline $\begin{array}{l}\text { F. oxysporum f. } \\
\text { sp. lycopersici }\end{array}$ & $\begin{array}{l}\text { Punica } \\
\text { granatum }\end{array}$ & Peel & Water & $\begin{array}{l}\text { Punicalagins and } \\
\text { ellagic acids }\end{array}$ & $\begin{array}{c}83 \% \text { mycelial } \\
\text { inhibition at } 0.5 \% \\
(w / v) \text { purified } \\
\text { extract } \\
\text { concentration }\end{array}$ & $\begin{array}{l}\text { Soil treatment with } \\
0.5 \%(w / w) \text { extract } \\
\text { concentration } \\
\text { reduced disease } \\
\text { incidence to half }\end{array}$ & [16] \\
\hline $\begin{array}{l}\text { F. oxysporum } \mathrm{f} . \\
\text { sp. radicis } \\
\text { lycopersici }\end{array}$ & $\begin{array}{c}\text { Solanum } \\
\text { linnaeanum }\end{array}$ & Leaf & Water & Not available & $\begin{array}{c}61 \% \text { mycelial } \\
\text { inhibition at } 4 \% \\
(v / v) \text { extract } \\
\text { concentration }\end{array}$ & $\begin{array}{l}\text { Substrate drench at } \\
25 \mathrm{~mL} / \text { seedling with } \\
30 \%(w / v) \text { extract } \\
\text { concentration } \\
\text { reduced leaf \& root } \\
\text { damage and vascular } \\
\text { discoloration by } \\
92.30 \% \text { and } 97.56 \% \text {, } \\
\text { respectively }\end{array}$ & [31] \\
\hline $\begin{array}{l}\text { F. oxysporum f. } \\
\text { sp. radicis- } \\
\text { lycopersici }\end{array}$ & $\begin{array}{l}\text { Lycium } \\
\text { arabicum }\end{array}$ & Leaf & $\begin{array}{l}\text { Distilled } \\
\text { water }\end{array}$ & Not available & $\begin{array}{c}33.5 \% \text { mycelial } \\
\text { inhibition at } 4 \% \\
(v / v) \text { extract } \\
\text { concentration }\end{array}$ & $\begin{array}{l}\text { Soil drenched with } \\
25 \mathrm{~mL} \text { of } 30 \%(v / v) \\
\text { extract concentration } \\
\text { reduced disease } \\
\text { symptoms by } 84.6 \%\end{array}$ & [32] \\
\hline $\begin{array}{l}\text { F. oxysporum f. } \\
\text { sp. lycopersici } \\
\text { race } 3\end{array}$ & $\begin{array}{l}\text { Ocimum } \\
\text { basilicum }\end{array}$ & $\begin{array}{l}\text { Leaves and } \\
\text { flowers }\end{array}$ & Water & Not available & Not available & $\begin{array}{l}\text { Seed soaked in } 20 \% \\
\text { aqueous extract for } \\
10 \mathrm{~h} \text { reduced disease } \\
\text { incidence to } 18 \% \text { as } \\
\text { compared to } 94.7 \% \\
\text { in control }\end{array}$ & {$[33]$} \\
\hline $\begin{array}{l}\text { F. oxysporum } \mathrm{f} . \\
\text { sp. lycopersici }\end{array}$ & $\begin{array}{l}\text { Moringa } \\
\text { oleifera }\end{array}$ & Leaves & Methanol & Not available & $\begin{array}{c}21 \% \text { reduction in } \\
\text { mycelial growth at } \\
4 \mathrm{~g} / \mathrm{mL} \\
\text { concentration }\end{array}$ & $\begin{array}{l}\text { Soil treatment with } \\
250 \mathrm{~mL} \text { of } 4 \mathrm{~g} / \mathrm{mL} \\
\text { extract concentration } \\
\text { significantly reduced } \\
\text { disease symptoms }\end{array}$ & [19] \\
\hline $\begin{array}{l}\text { F. oxysporum f. } \\
\text { sp. lycopersici }\end{array}$ & $\begin{array}{c}\text { Theobroma } \\
\text { cacao }\end{array}$ & Pod husk & $\begin{array}{c}\text { Acetone: } \\
\text { Water (7:3) }\end{array}$ & Not available & Not available & $\begin{array}{l}100 \mathrm{~mL} \text { of } 8 \%(v / v) \\
\text { extract formulation } \\
\text { per plant reduced } \\
\text { wilt incidence to } \\
23.8 \% \text { compared to } \\
100 \% \text { in control }\end{array}$ & [20] \\
\hline \multirow{2}{*}{ F. oxysporum } & $\begin{array}{l}\text { Juglans } \\
\text { microcarpa }\end{array}$ & Leaf & Ethanol & $\begin{array}{c}\text { Vitamin E } \\
\text { acetate, Phytol, } \\
\text { Benze- } \\
\text { neethanamine, }\end{array}$ & Not available & $\begin{array}{l}\text { Root treatment with } \\
5000 \mathrm{mg} / \mathrm{L} \text { extract } \\
\text { concentration } \\
\text { reduced disease } \\
\text { incidence to } 37.5 \%\end{array}$ & \multirow{2}{*}{ [17] } \\
\hline & Juglans mollis & Leaf & Ethanol & $\begin{array}{l}\text { Hexanedioic acid } \\
\text { dioctyl ester, } \\
\text { Hexadecanoic } \\
\text { acid, ethyl ester, }\end{array}$ & Not available & -do- & \\
\hline
\end{tabular}


Table 1. Cont.

\begin{tabular}{|c|c|c|c|c|c|c|c|}
\hline Pathogen & Source Plant & Plant Part & Solvent & $\begin{array}{l}\text { Major Bioactive } \\
\text { Compounds }\end{array}$ & In-Vitro Control & $\begin{array}{c}\text { In Vivo Disease } \\
\text { Control }\end{array}$ & Reference \\
\hline F. oxysporum & $\begin{array}{l}\text { Stevia } \\
\text { rebaudiana }\end{array}$ & Leaf & Hexane & Austroinulin & $\begin{array}{l}54.9 \% \text { mycelial } \\
\text { inhibition at } \\
833 \text { ppm extract } \\
\text { concentration }\end{array}$ & $\begin{array}{l}\text { Substrate treatment } \\
\text { with } 3 \text { mL of } \\
500 \text { ppm extract } \\
\text { caused a reduction in } \\
\text { stunting incidences }\end{array}$ & [21] \\
\hline $\begin{array}{l}\text { F. oxysporum f. } \\
\text { sp. lycopersici }\end{array}$ & $\begin{array}{l}\text { Pistacia } \\
\text { lentiscus }\end{array}$ & Leaf & Water & $\begin{array}{c}\text { Quercetin, } \\
\text { Protocatechuic } \\
\text { acid, } \\
\text { Chlorogenic acid }\end{array}$ & $\begin{array}{c}82.40 \% \text { mycelial } \\
\text { inhibition at } 5 \% \\
(v / v) \text { extract } \\
\text { concentration }\end{array}$ & $\begin{array}{c}29.17 \% \text { disease } \\
\text { incidence in } \\
\text { treatment as } \\
\text { compared to } 83.33 \% \\
\text { in untreated control } \\
\text { when treatment was } \\
\text { done using } 100 \% \\
\text { extract }\end{array}$ & [18] \\
\hline R. solani & $\begin{array}{l}\text { Euphorbia } \\
\quad \text { hirta }\end{array}$ & Leaf & $70 \%$ Ethanol & $\begin{array}{c}\text { Phenols, } \\
\text { alkaloids, and } \\
\text { polysaccharides }\end{array}$ & $\begin{array}{l}100 \% \text { mycelial } \\
\text { inhibition at } \\
10 \mathrm{mg} / \mathrm{mL} \\
\text { concentration }\end{array}$ & $\begin{array}{l}\text { Spray treatment with } \\
2.50 \mathrm{mg} / \mathrm{mL} \text { extract } \\
\text { concentration } \\
\text { reduced disease } \\
\text { incidence by } 29.24 \%\end{array}$ & [34] \\
\hline \multirow{3}{*}{$\begin{array}{l}\text { F. oxysporum } \mathrm{f} . \\
\text { sp. lycopersici }\end{array}$} & $\begin{array}{l}\text { Allium } \\
\text { satioum }\end{array}$ & Cloves & Water & Not available & Not available & $\begin{array}{l}\text { Spray treatment } \\
\text { reduced disease } \\
\text { incidence by } 8.40 \% \\
\text { compared to } 84.46 \% \\
\text { in control }\end{array}$ & \multirow{3}{*}{ [35] } \\
\hline & $\begin{array}{l}\text { Azadirachta } \\
\text { indica }\end{array}$ & Leaf & Water & Not available & Not available & $\begin{array}{l}\text { Spray treatment } \\
\text { reduced disease } \\
\text { incidence by } 10.70 \% \\
\text { compared to } 84.46 \% \\
\text { in control }\end{array}$ & \\
\hline & $\begin{array}{l}\text { Zingiber } \\
\text { officinale }\end{array}$ & Rhizome & Water & Not available & Not available & $\begin{array}{l}\text { Spray treatment } \\
\text { reduced disease } \\
\text { incidence by } 11.90 \% \\
\text { compared to } 84.46 \% \\
\text { in control }\end{array}$ & \\
\hline V. dahliae & $\begin{array}{c}\text { Allium cepa } \\
\text { var. } \\
\text { aggregatum }\end{array}$ & Root exudate & $\begin{array}{l}\text { Deionized } \\
\text { water }\end{array}$ & Not available & $\begin{array}{l}0.1 \mathrm{~g} / \mathrm{mL} \text { extract } \\
\text { concentration } \\
\text { mixed with media } \\
(1: 1) \text { caused } \\
\text { significant } \\
\text { reduction in } \\
\text { mycelial biomass }\end{array}$ & Not available & [36] \\
\hline
\end{tabular}

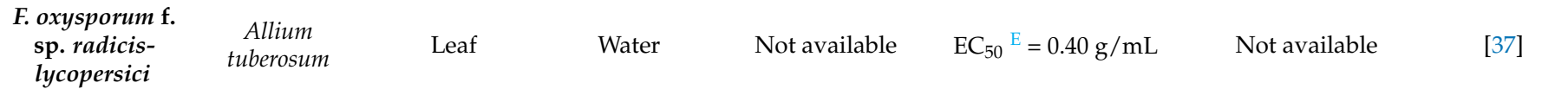

\begin{tabular}{|c|c|c|c|c|c|c|c|}
\hline P. debaryanum & $\begin{array}{c}\text { Aegle } \\
\text { marmelos }\end{array}$ & Leaf & Methanol & Not available & $\begin{array}{c}100 \% \text { inhibition at } \\
1000 \mu \mathrm{L} \text { extract } \\
\text { concentration }\end{array}$ & $\begin{array}{l}\text { Soil treatment with } \\
4 \% \text { extract } \\
\text { concentration } \\
\text { reduced pre and } \\
\text { post-emergence } \\
\text { damping-off } \\
\text { incidences to } 16.22 \% \\
\text { and } 34.67 \% \text { as } \\
\text { compared to } 35.90 \% \\
\text { and } 42.67 \% \text { in } \\
\text { control, respectively }\end{array}$ & [38] \\
\hline $\begin{array}{l}\text { F. oxysporum f. } \\
\text { sp. lycopersici }\end{array}$ & Rhus muelleri & Leaf & Ethanol & $\begin{array}{c}\text { Ethyl } \\
\text { isoallocholate, } \\
7,8- \\
\text { epoxylanostan- } \\
\text { 11-ol, } \\
\text { 3-acetoxy }\end{array}$ & $\begin{array}{c}\mathrm{MIC}_{50}=3363 \mathrm{ppm} \\
\mathrm{MIC}_{90} \mathrm{~F}= \\
11,793 \mathrm{ppm}\end{array}$ & Not available & [39] \\
\hline
\end{tabular}


Table 1. Cont.

\begin{tabular}{|c|c|c|c|c|c|c|c|}
\hline Pathogen & Source Plant & Plant Part & Solvent & $\begin{array}{l}\text { Major Bioactive } \\
\text { Compounds }\end{array}$ & In-Vitro Control & $\begin{array}{c}\text { In Vivo Disease } \\
\text { Control }\end{array}$ & Reference \\
\hline \multirow{2}{*}{ R. solani } & $\begin{array}{l}\text { Euphorbia } \\
\quad \text { hirta }\end{array}$ & Leaf & $70 \%$ ethanol & $\begin{array}{c}\text { Hydroxycinnamic } \\
\text { acids, } \\
\text { Hydroxybenzoic } \\
\text { acids, } \\
\text { Isocoumarins, } \\
\text { Elagitannins }\end{array}$ & $\mathrm{IC}_{50}=3.66 \mathrm{mg} / \mathrm{mL}$ & Not available & \multirow{2}{*}{ [27] } \\
\hline & -do- & -do- & Water & $\begin{array}{c}\text { Gallotannins, } \\
\text { Hydroxybenzoic } \\
\text { acids, Hydrox- } \\
\text { ycinnamic acids, } \\
\text { Flavonols }\end{array}$ & $\begin{array}{c}\mathrm{IC}_{50}= \\
32.14 \mathrm{mg} / \mathrm{mL}\end{array}$ & Not available & \\
\hline $\begin{array}{l}\text { F. oxysporum f. } \\
\text { sp. lycopersici; } \\
\quad \text { F. solani }\end{array}$ & $\begin{array}{l}\text { Allium } \\
\text { satioum }\end{array}$ & Bulb & Water & $\begin{array}{c}\text { Flavanoid, } \\
\text { terpenoid, } \\
\text { saponin, steroids, } \\
\text { tannins, cardiac } \\
\text { glycoside, } \\
\text { coumarins }\end{array}$ & $\begin{array}{l}100 \% \text { mycelial } \\
\text { growth inhibition } \\
\text { at } 8 \% \text { extract } \\
\text { concentration }\end{array}$ & Not available & [40] \\
\hline P. ultimum & $\begin{array}{l}\text { Curcuma } \\
\text { longa }\end{array}$ & Rhizome & 95\% Ethanol & Not available & $\begin{array}{c}55.6 \% \text { mycelial } \\
\text { inhibition at } 2 \% \\
(v / v) \text { extract } \\
\text { concentration }\end{array}$ & Not available & [41] \\
\hline $\begin{array}{l}\text { F. oxysporum f. } \\
\text { sp. lycopersici }\end{array}$ & $\begin{array}{l}\text { Cenchrus pen- } \\
\text { nisetiformis }\end{array}$ & Shoot & $\begin{array}{l}\text { Ethyl acetate } \\
\text { sub-fraction } \\
\text { of methanol } \\
\text { extract }\end{array}$ & $\begin{array}{c}\text { Hexadecanoic } \\
\text { acid, ethyl-ester, } \\
\text { Phenol, } \\
\text { 2,4-bis }\{1,1- \\
\text { dimethlethyl\}- }\end{array}$ & $\begin{array}{l}100 \% \text { decline in } \\
\text { fungal biomass } \\
\text { production at } \\
12.5 \mathrm{mg} / \mathrm{mL} \\
\text { concentration }\end{array}$ & Not available & [42] \\
\hline S. rolfsii & $\begin{array}{l}\text { Ocimum } \\
\text { basilicum }\end{array}$ & Leaf & Water & Not available & $\begin{array}{c}33.35 \% \text { reduction } \\
\text { in mycelial growth } \\
\text { at } 100 \% \\
\text { concentration }\end{array}$ & $\begin{array}{l}\text { Soil drenching with } \\
100 \mathrm{~mL} \text { of } 100 \% \\
\text { extract concentration } \\
\text { reduced damping-off } \\
\text { incidences by } 30 \%\end{array}$ & [43] \\
\hline
\end{tabular}

A Minimum Inhibitory Concentration needed to inhibit $50 \%$ of the living process; ${ }^{\mathrm{B}}$ Substance concentration at which only half of its maximum inhibitory effect is observed; ${ }^{C}$ Minimum Inhibitory Concentration; ${ }^{D}$ Minimum fungicidal concentration; ${ }^{E}$ Substance concentration at which only half of its maximum effect is observed; F Minimum Inhibitory Concentration needed to inhibit $90 \%$ of the living process.

Though prospective PSMs are infrequently utilised to manage soil-borne infections in chilli plants compared to tomatoes, their application has improved significantly in recent years (Table 2). Wang et al. [44] studied the efficacy of cuminic acid, a pure compound extracted from the seeds of Cuminum cyminum against Phytophthora capsici. Cuminic acid at $1000 \mu \mathrm{g} / \mathrm{mL}$ concentration proved to be quite efficacious in managing the disease and exhibited $70.89 \%$ disease control. Methanolic extracts of Boerhavia diffusa roots at $1 \%$ concentration significantly reduced the diseases symptoms caused by $\mathrm{Ph}$. Capsici in chilli plants [45]. At a concentration of 100 ppm, Cymbopogon citratus essential oil demonstrated a $60.5 \%$ reduction in disease severity, which confirmed its effectiveness against Ph. Capsici [46]. A study by Pandey et al. [47] concluded that aqueous extract of Lantana camara leaves is particularly efficient in managing pre- and post-emergence damping-off caused by P. aphanidermatum. Seed treatment with the Glycyrrhiza uralensis root extracts greatly reduced the seedling mortality caused by P. aphanidermatum and $R$. solani [48]. 
Table 2. PDPs reported to control soil-borne diseases in chilli.

\begin{tabular}{|c|c|c|c|c|c|c|c|}
\hline Pathogen & Source Plant & Plant Part & Solvent & $\begin{array}{l}\text { Major Bioactive } \\
\text { Compounds }\end{array}$ & In-Vitro Control & $\begin{array}{l}\text { In-Vivo Disease } \\
\text { Control }\end{array}$ & Reference \\
\hline \multicolumn{8}{|c|}{ Pure compound } \\
\hline Ph. capsici & $\begin{array}{l}\text { Cuminum } \\
\text { cyminum }\end{array}$ & Seed & $\begin{array}{c}\text { Not } \\
\text { available }\end{array}$ & Cuminic acid & $\begin{array}{c}\mathrm{EC}_{50}(\text { Mycelial } \\
\text { growth })=14.54 \pm \\
5.23 \mu \mathrm{g} / \mathrm{mL} ; \\
\mathrm{EC}_{50} \text { (Zoospore } \\
\text { germination) }=6.97 \\
\pm 2.82 \mu \mathrm{g} / \mathrm{mL}\end{array}$ & $\begin{array}{c}\text { Irrigation with } 10 \mathrm{~mL} \\
\text { of } 1000 \mu \mathrm{g} / \mathrm{mL} \\
\text { compound } \\
\text { concentration exhibited } \\
70.89 \% \text { disease control } \\
\text { efficacy }\end{array}$ & [44] \\
\hline \multicolumn{8}{|l|}{ Essential oil } \\
\hline Ph. capsici & $\begin{array}{l}\text { Cymbopogon } \\
\text { citratus }\end{array}$ & Leaf & - & $\begin{array}{l}\text { z-citral, } \beta \text {-geranial, } \\
\text { caryophyllene }\end{array}$ & $\mathrm{EC}_{50}=31.473 \mathrm{ppm}$ & $\begin{array}{c}\text { Soil drenching with } \\
50 \mathrm{~mL} \text { of } 100 \mathrm{ppm} \text { oil } \\
\text { concentration reduced } \\
\text { disease severity by } \\
60.5 \%\end{array}$ & [46] \\
\hline Ph. capsici & $\begin{array}{l}\text { Eupatorium } \\
\text { adenophorum }\end{array}$ & Leaf & - & $\begin{array}{l}\text { OA (9-oxo- } \\
\text { agerophorone), } \\
\text { ODA (9-oxo-10, } \\
\text { 11-dehydro- } \\
\text { agerophorone) }\end{array}$ & $\mathrm{MIC}=500 \mu \mathrm{g} / \mathrm{mL}$ & Not available & [49] \\
\hline $\begin{array}{c}F . \\
\text { oxysporum }\end{array}$ & $\begin{array}{l}\text { Syzygium } \\
\text { aromaticum }\end{array}$ & $\begin{array}{c}\text { Not } \\
\text { available }\end{array}$ & - & Eugenol & $\mathrm{MIC}=0.25 \%(w / v)$ & $\begin{array}{l}\text { Seedling treatment } \\
\text { with } 0.5 \%(w / v) \\
\text { essential oil } \\
\text { concentration reduced } \\
\text { disease severity index } \\
\text { to } 56.20 \% \text { compared to } \\
100 \% \text { in control in } \\
\text { greenhouse }\end{array}$ & [50] \\
\hline \multicolumn{8}{|l|}{ Plant extract } \\
\hline Ph. capsici & $\begin{array}{l}\text { Boerhavia } \\
\text { diffusa }\end{array}$ & Root & Methanol & Not available & $\mathrm{MIC}=0.5 \%$ & $\begin{array}{c}1 \% \text { plant extract } \\
\text { concentration at } \\
6 \mathrm{~mL} / \text { plant reduced } \\
\text { disease symptoms } \\
\text { significantly }\end{array}$ & [45] \\
\hline $\begin{array}{l}\text { P. aphanider- } \\
\text { matum }\end{array}$ & $\begin{array}{l}\text { Lantana } \\
\text { camara }\end{array}$ & Leaf & Water & Not available & Not available & $\begin{array}{l}\text { Seed treatment reduced } \\
\text { pre-emergence and } \\
\text { post-emergence } \\
\text { damping-off incidences } \\
\text { to } 7.08 \% \text { and } 10.31 \% \text { as } \\
\text { compared to } 40 \% \text { and } \\
62.32 \% \text { in control }\end{array}$ & [47] \\
\hline $\begin{array}{l}\text { P. aphanider- } \\
\text { matum }\end{array}$ & $\begin{array}{l}\text { Glycyrrhiza } \\
\text { uralensis }\end{array}$ & Root & $\begin{array}{l}\text { Ethyl } \\
\text { acetate } \\
\text { subfrac- } \\
\text { tion of } 80 \% \\
\text { methanol } \\
\text { extract }\end{array}$ & Not available & $\begin{array}{l}62.6 \% \text { mycelial } \\
\text { inhibition at } 10 \\
\mu \mathrm{g} / \mathrm{mL} \text { extract } \\
\text { concentration }\end{array}$ & $\begin{array}{l}\text { Seed treatment resulted } \\
\text { in } 82 \% \text { seed } \\
\text { germination and } \\
21.95 \% \text { seedling } \\
\text { mortality as compared } \\
\text { to } 50 \% \text { and } 96 \% \text { in } \\
\text { control }\end{array}$ & \multirow{2}{*}{ [48] } \\
\hline R. solani & -do- & -do- & -do- & Not available & $\begin{array}{l}77.6 \% \text { mycelial } \\
\text { inhibition at } 10 \\
\mu \mathrm{g} / \mathrm{mL} \text { extract } \\
\text { concentration }\end{array}$ & $\begin{array}{c}\text { Seed treatment resulted } \\
\text { in } 88 \% \text { seed } \\
\text { germination and } \\
13.63 \% \text { seedling } \\
\text { mortality as compared } \\
\text { to } 54 \% \text { and } 85.18 \% \text { in } \\
\text { control }\end{array}$ & \\
\hline Ph. capsici & $\begin{array}{l}\text { Helianthus } \\
\text { tuberosus }\end{array}$ & Leaf & $\begin{array}{l}\text { n-Butanol } \\
\text { fraction of } \\
70 \% \\
\text { ethanol } \\
\text { extract }\end{array}$ & $\begin{array}{c}\text { Methyl quercetin } \\
\text { glycoside (MQG) } \\
\text { Caffeoylquinic acid } \\
\text { isomer }\end{array}$ & $\mathrm{IC}_{50}=0.839 \mathrm{~g} / \mathrm{L}$ & Not available & [51] \\
\hline
\end{tabular}


The bioactive metabolites in the plant parts of certain species have been discovered to have a significant pesticidal value (Figure 2). While the pesticidal effects of purified metabolites are exploited in a variety of crops [52-54], we discovered very little research on tomatoes and chilli peppers. Smaili et al. [25,26], in their study, used derivatives of the $\alpha$-euphorbol, 31-norlanostenol, and lupeol acetate compounds isolated from the latex of different Euphorbia spp. as a seed treatment and spray for the control of Verticillium dahliae in tomato, and observed a significant reduction in symptom development at a very low concentration of $10 \mu \mathrm{g} / \mathrm{mL}$. The derivatives of these compounds worked as the elicitors of plant defense. Wang et al. [44] studied the efficacy of cuminic acid, a pure compound extracted from the seeds of Cuminum cyminum against Phytophthora capsici. Cuminic acid, other than exhibiting significant inhibition of mycelia and zoospore germination, proved to be quite efficacious at $1000 \mu \mathrm{g} / \mathrm{mL}$ concentration in managing the disease and exhibited $70.89 \%$ disease control. Even though purified compounds are highly effective, utilising them is an expensive affair due to the cost of compound isolation/purification and recovery of a minute proportion of the component.

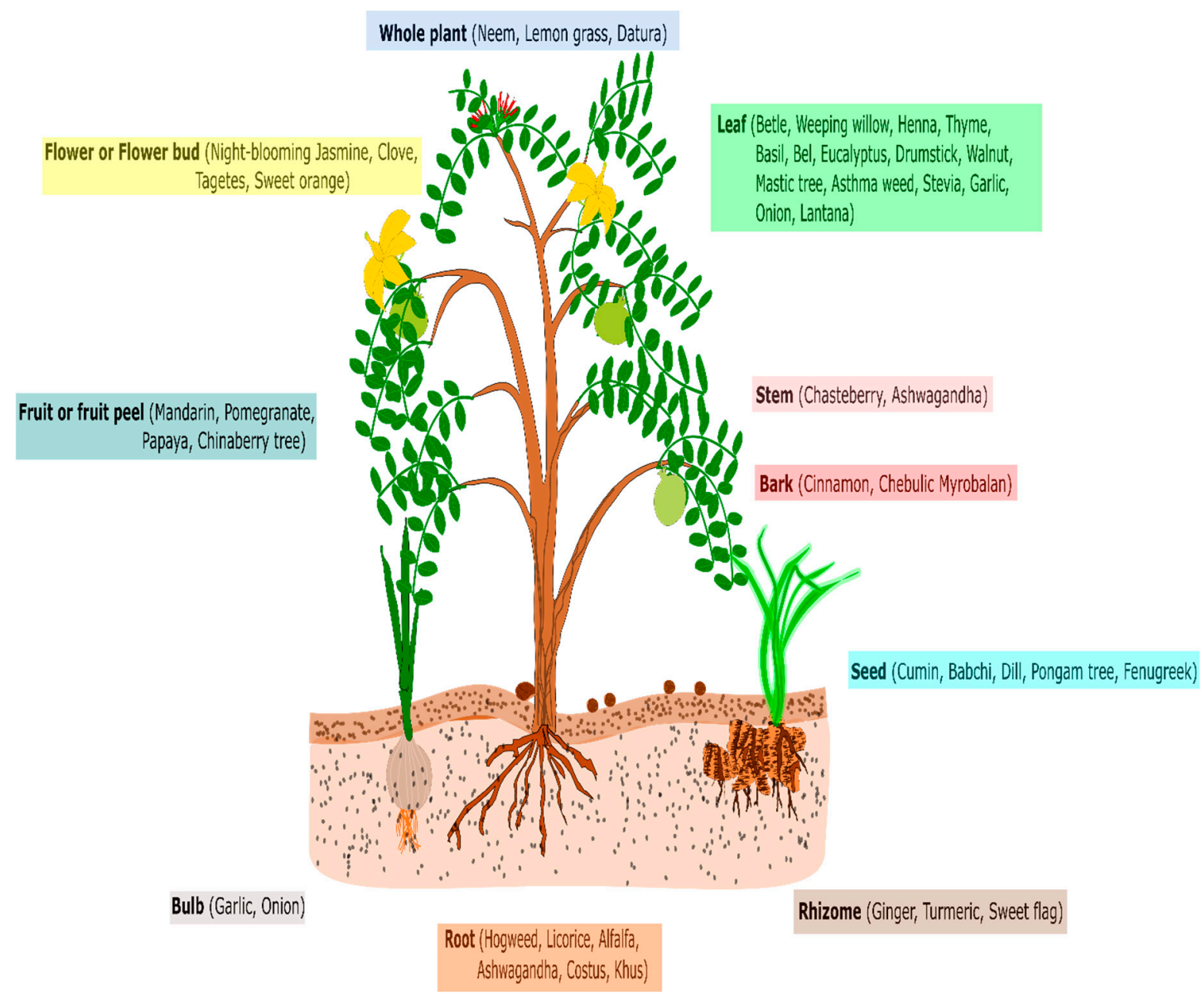

Figure 2. Parts of known plant species rich with bioactive compounds.

\subsection{Using Total Phytobiomass (Land to Land Approach)}

Studies on the efficacy of PDPs against phytopathogens primarily aim to develop them as fungicides. Using these bioactive-rich plants as green manure or companion plants provides an efficient alternative, harnessing their allelopathic capabilities. Brassicaceae crops used as soil biofumigant have garnered considerable interest as an alternative to methyl bromide. The hydrolysis of sulfur and nitrogen-containing glucosinolates (GLS) 
found in plants from the Brassicaceae family into extremely toxic volatile isothiocyanates is triggered by the enzyme myrosinase in the presence of water [55]. In an in-vitro experiment by Pane et al. [56], volatiles obtained from Brassica carinata seed meal (BCSM) showed dose-dependent growth inhibition of F. oxysporum f. sp. lycopersici. Complete growth inhibition was obtained at $100 \mathrm{mg} / \mathrm{mL}$ concentration. Also, when combined with thyme oil and Bacillus amyloliquefaciens, the volatiles inhibited growth up to $34 \%$ more than the bacterial antagonist alone. The use of BCSM at a rate of $0.5 \mathrm{~g} /$ pot reduced the incidence and severity of Fusarium wilt in tomato plants by around 50\%. According to Ma et al. [57], seed meal amendments affected the fungal mycobiota of the soil. They observed an increase in the Fusarium, Hypocreales, and Chaetomium populations when they employed Camelina sativa seed meal as an amendment due to the increased nutrient availability.

Al-Hammouri et al. [58] found that the root-associated R. solani of chilli reduced when they amended the soil with Calligonum aboveground plant parts and olive leaves. As described in the literature, the principal active chemicals in olive leaves include phenols such as oleuropein and hydroxytyrosol. Besides, it was thought that the soil microbiome would change for the better and help the chilli plants. Kadoglidou et al. [12] used spearmint and Greek oregano aboveground plant parts as soil amendments at $4 \%(w / w)$ against soilborne diseases (Fusarium and Vertcillium wilt) of tomato plants. After amendments, GC/MS examination of soil samples revealed a decrease in monoterpenes and an increase in sesquiterpenes. Growing parameters improved due to the long-term persistence of plant-growth-promoting sesquiterpenes, oxygenated monoterpenes such as carvacrol in the soil, along with high microbial activity, due to degradation of plant material. Even after 50 days, the tomato plant showed no signs of Fusarium or Verticillium wilt.

\section{Antifungal Screening Assays of PDPs}

When it comes to evaluating the antifungal potential of PDPs (pure metabolite or in crude form as extracts or essential oils), screening assays are unmatched, as they aid in labelling them as entitled and qualified for further advancement. The following subsections will detail the typical screening procedures utilised and a potential alternative to these conventional approaches.

\subsection{Conventional Approach}

For starting to narrow down possible antifungal PDPs, there are three types of broad classification. This classification includes dilution methods, diffusion methods, and bioautographic procedures. Well-known dilution methods are agar and broth dilutions, wherein the culture medium is mixed with the test sample(s) and inoculated with the target pathogen. Additionally, macro- or micro-dilution techniques aid in determining the minimal inhibitory and fungicidal concentrations of the test sample $[59,60]$. The diffusion methods involve well diffusion, disc diffusion, and poison food techniques [60]. However, diffusion methods are often inadequate to reveal the actual antifungal activity because of the meager diffusion rate of hydrophobic bioactive metabolites through agar media. In bioautographic methods, the use of thin-layer chromatography (TLC) precedes the antifungal assay. The bioautographic methods can be divided into contact, direct, and immersion bioautography [61]. Despite their widespread use, these conventional procedures frequently fall short due to low reproducibility and other restrictions such as higher cost, sluggishness, and arduousness.

\subsection{In Silico Approach}

Thanks to the development of bioinformatics, scientists can use computer-aided technologies to screen antifungal compounds with knowledge obtained from genomic sequencing of the pathogens and computational work on the structures of antifungal compounds and the biological targets $[62,63]$. Such an in-silico approach has aided in the rapid screening of several antifungal chemicals. These computationally selected, natural antifungal chemicals rely on their binding affinity with the targeted virulent compounds. 
Virulence proteins involved in sterol, chitin, melanin, tubulin, and protein biosynthesis have been the target of interest for synthetic fungicides [64]. Researchers have shown interest in targeting virulence proteins linked to sterol, chitin, melanin, and tubulin biosynthesis [64]. Pathogenesis-related genes and their translational products involved in the cell wall or membrane degradation, melanin biosynthesis, phytotoxin synthesis, effector protein synthesis, and fungal cells growth/differentiation have been the major targets for these computational based studies [65-68]. The different processes of biopesticide design are illustrated in Figure 3. The 3D structure of required binding molecules can be obtained using special databases such as PubChem and ChEMBL [69,70].

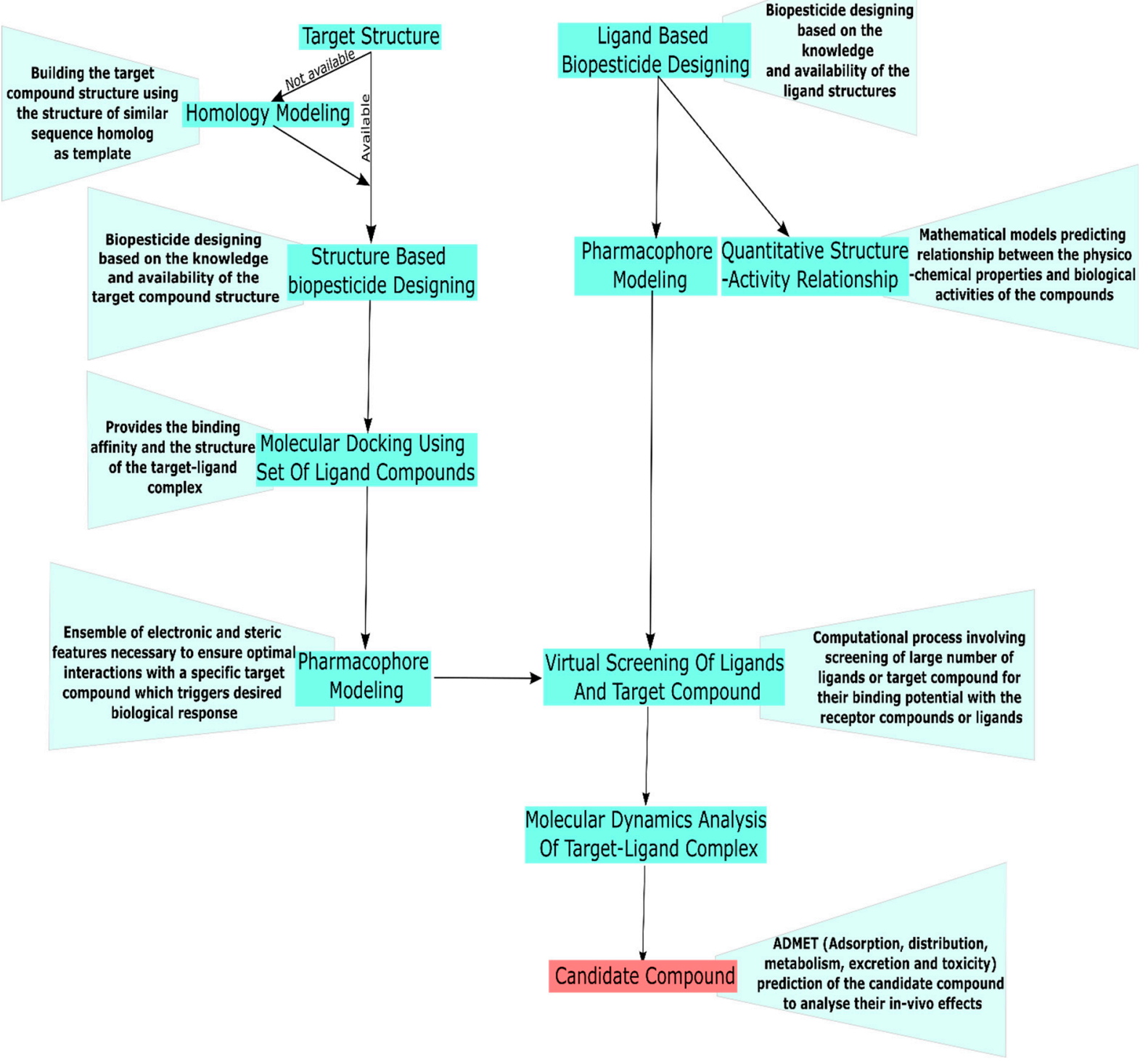

Figure 3. Schematic view of the steps involved in candidate compound identification and design (in silico approach).

Silva et al. [71] targeted the $\beta$-glucosidases (FsBglc) protein of F. solani f. sp. piperis, which have a critical role in host cell wall degradation. The 3D structure for the FsBglc protein was prepared using the $\beta$-glucosidases enzyme from Kluyveromyces marxianus (KmBglI) as a template through homology modelling. The ligand molecules eugenol and methyl eugenol showed negative MolDock scores and $\mathrm{H}$ bond energy when interacted with FsBg1c protein. The presence of many hydroxyl groups and a benzene ring in eugenol and methyl 
eugenol disrupted the hydrogen bonding network, resulting in negative H-bond energy and MolDock scores of these ligand molecules. The in-vitro and in-vivo control of Alternaria alternata by Anadenanthera colubrina methanol extract, for some part, was attributed to the presence of $\beta$-sitosterol and $\beta$-sitosteryl linoleate by Campos et al. [72]. Both of these bioactive compounds, which share some structural similarities with ergosterol, may interfere with the synthesis or function of ergosterol in the fungal cell. The in silico studies have confirmed the binding affinity of these two ligands with the oxysterol-binding proteins of Saccharomyces cerevisiae, depriving ergosterol of its role in fungus. Priyadharsini et al. [73] targeted the melanin biosynthesis disruption by inhibiting the scytalone dehydratase (SCD) activity in their quest to control the Colletotrichum lagenarium. The 20 phytochemical compounds were assessed for their binding potential with the SCD active site residues. Five compounds viz., atalaphylline, licochalcone A, vitrofolal F, gingerol, and buxifoliadine exhibited even higher binding affinity than the synthetic fungicide carpropamid. Trichothecene mycotoxins produced by the Fusarium species have been a reason for cereal crop losses due to their phytotoxic effects. Pani et al. [74] scrutinised a chemical that mimicked the natural substrate of trichodiene synthase (TRI5), farnesyl pyrophosphate, to combat the effects of F. culmorum. The best inhibitors of trichothecene production were me-dehydrozingerone, propyl gallate, magnolol, and eugenol dimers.

\section{Hurdles in Bringing Pest-Protection Research to Market}

While PDPs have been touted as a safer and perhaps more effective alternative to synthetic pesticides, we need to delve deeper into the reasons behind their lack of market acceptance. The previous section briefly described various databases that include information on the structural properties of secondary plant metabolites. More than 200,000 plant secondary metabolites have been found, which is remarkable [75]. Additionally, a bibliographical search of the Scopus database was undertaken between 2000 and 2021 . We performed the search by using different combinations of keywords: essential oil, plant extract, plant metabolite, plant secondary metabolite, plant bioactive compound, botanical, fungicide, antifungal, fungitoxic, bactericidal, bactriostatic, antiviral, formulation, emulsion, and suspension. Papers found through this search were restricted to the "articles" category. It is critical to recognise that this bibliographic search of relevant studies does not necessarily cover all research articles on the subject. The investigation has determined that over 14,500 published papers have been completed that examine the capability of various plant extracts, essential oils, and active metabolites to control plant disease pathogens (e.g., fungi, bacteria, and viruses) (Figure 4). Approximately 1300 investigations attempted to formulate the identified and extracted active chemicals into a useable form within the same period.

Furthermore, only 665 published patents for the relevant domain were discovered by patent mining in the Espacenet databases. The keywords used in the patent search were: fungicide, antifungal, fungitoxic, bactericidal, bactriostatic, antiviral, formulation, emulsion, and suspension in the IPC class description "A01N65/00". This observed gap between recognising prospective chemicals and developing them into a functional product demonstrates the significant bridge that we must build between the two. Isman [76] argues the necessity of converting already-established botanicals into useable forms, as opposed to accumulating knowledge through isolating and discovering an increasing number of potential molecules. Though this is the case, most research on PDPs has been dedicated to controlling insects (and not phytopathogens) in farm areas and storage facilities [5]. Rather than a lack of scientific breakthroughs, we argue that there are alternative variables for the enigma of PDP commercialisation limitations. 

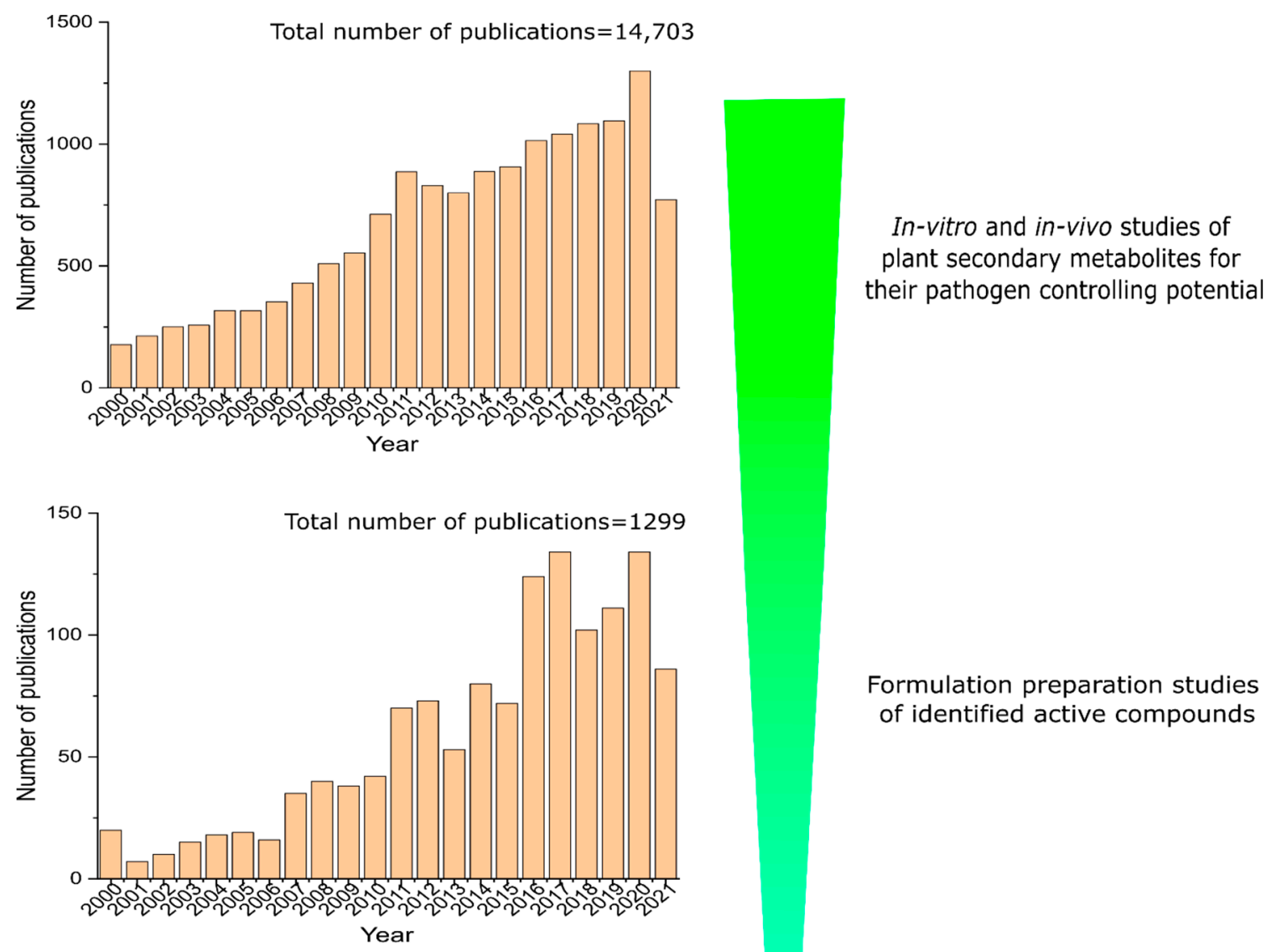

their pathogen controlling potential

Formulation preparation studies of identified active compounds

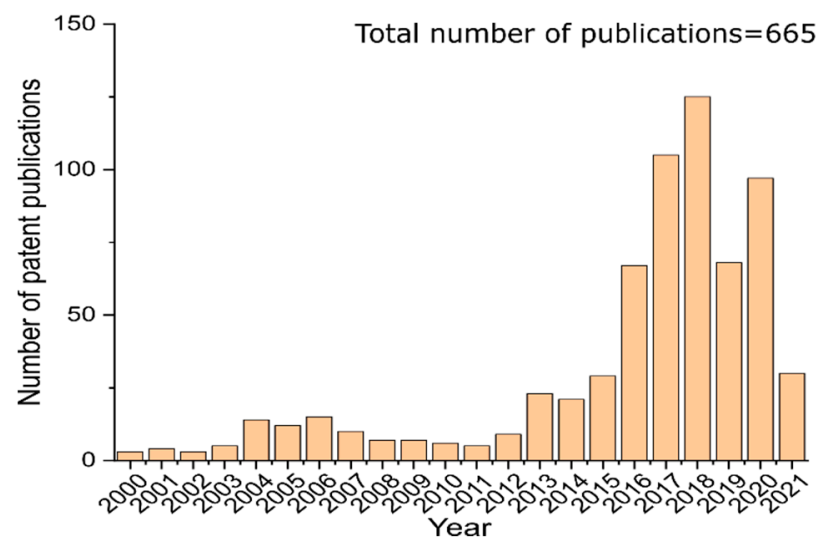

Published patents with commercial prospects

Figure 4. Number of research publications for anti-phytopathogenic of PSMs and formulation development in Scopus database from 2000 to 2021; Number of patent publications in Espacenet patent database from 2000 to 2021.

Secondary metabolites that have been discovered to fight plant diseases appear in a significant number of high-impact papers. Academic and research institutes (mainly from developing countries) lack the backing from industry or the federal government, which is needed to cover the cost of conducting research and development, which is why few products have been developed $[77,78]$. This is the reason why most of them are content with publishing or patenting their discoveries. Many big manufacturers have shied away from the PDPs market because of its reputation for being highly focused on targeting specific pests [79]. Industries are more likely to build a product with massive market reach and widespread effect. As previously said, the process of isolating a novel green chemical and converting it into a marketable product is costly. As a result, industries are inclined toward the commercialisation of generic pesticides. Farmers would be the end-users of 
green pesticides, but perceptions of them, such as lower effectiveness, lower productivity, and high cost of these green alternatives, have been observed as the cause of reluctance in their use [80]. Thus, it is a vicious cycle in which a lack of intents in all stakeholders undermines the chance of a thriving green pesticides sector. Farmers have developed a reliance on generic pesticides since they are inexpensive and have been used for decades, while manufacturers have virtually ceased innovation due to a lack of demand and market for green alternatives.

Regulatory policies have also impeded the commercialisation of PDPs. The European Union (EU) still has stringent rules for the registration of botanical-based pesticides, where they fall under the same regulatory framework as chemical pesticides [81]. Japan has not framed a separate set of regulations for the botanical compounds, which, in principle, require them to be evaluated similarly to chemically synthesised substances. Countries such as Canada and the USA have recognised the fact that these natural substances cannot be evaluated using the framework developed for conventional chemical pesticides [82]. For biopesticides in India, a discrete set of information is necessary for different botanicals (e.g., Cymbopogon plant extract, Pyrethrum extract, Neem-based products, etc.), which makes the procedure somewhat onerous [83]. Despite the ease in the regulatory framework in some countries, the registration, and commercialisation of botanical compounds have fallen prey to the huge cost and prolonged procedures [76,84].

\section{Conclusions and Future Perspectives}

Tomato and chilli, two of the significant crops of the Solanaceae family, have been majorly impacted by soil-borne fungal diseases. For a long time, synthetic fungicides and fumigants have played a critical role in the management of these diseases. However, due to their repeated records of damaging off-target effects, eradication of them is being aimed for. PDPs with subsidiary effects along with their antifungal potential have become a reliable alternative. Many PDPs were found to be quite effective in managing the soil-borne fungal phytopathogens in chilli and tomato plants.

When used as soil amendments, other than suppressing the pathogen directly with the released metabolites, drastic changes in the beneficial soil microbiota were observed. The microbiome plays a crucial role in the plant-pathogen-microbiome tripartite interaction. So, a comprehensive evaluation of the effect of a particular botanical product or bioactive rich soil amendment on the soil microbiome would provide information about their effectiveness in actual field conditions.

In silico approaches can play a crucial role in assessing these active metabolites structural-activity relationships and for screening potential candidate compounds based on the affinity potential with the target proteins. However, the use of these approaches is far behind their actual potential.

This investigation documented the undeniable gap between research and marketing of PDPs. Regulatory impediments, costly R\&D processes to synthesise a new green molecule and later convert it to a product, a lack of commitment among stakeholders, and a lack of awareness among farmers for green options are all points of contention. Slow-release pesticides or nano-formulations of PDPs are viewed as the prospect of formulation technology in the agro-chemical sector. Having stated that, our investigation indicates that, notwithstanding the facts we uncovered, particular long-standing concerns demand resolution.

Author Contributions: Conceptualisation, A.S. and S.S.; writing-original draft preparation, H.A.; Supervision, A.S. and S.S.; writing — review and editing, A.S., P.P., S.S., F.F.H., A.G. and R.Z.S.; funding acquisition, P.P., F.F.H., A.G. and R.Z.S. All authors have read and agreed to the published version of the manuscript.

Funding: This work was supported by the University Grant Commission under the scheme of Junior Research Fellowship (UGC-JRF). Open access was supported by the Helsinki University Library, Finland. 
Institutional Review Board Statement: Not applicable.

Informed Consent Statement: Not applicable.

Data Availability Statement: All the data is present in the manuscript.

Acknowledgments: The authors are extremely thankful to the University Grant Commission (UGC), India, for providing financial assistance. P.P. thanks the support of the Eötvös Research Fund. Research fellowship granted by the Alexander von Humboldt Foundation, Bonn, Germany, to AG is also gratefully acknowledged.

Conflicts of Interest: The authors declare no conflict of interest.

\section{References}

1. Haq, I.U.; Sarwar, M.K.; Faraz, A.; Latif, M.Z. Synthetic chemicals: Major component of plant disease management. In Plant Disease Management Strategies for Sustainable Agriculture through Traditional and Modern Approaches; Haq, I.U., Ijaz, S., Eds.; Springer: Berlin/Heidelberg, Germany, 2020; pp. 53-81.

2. Chowdhary, K.; Kumar, A.; Sharma, S.; Pathak, R.; Jangir, M. Ocimum sp.: Source of biorational pesticides. Ind. Crops Prod. 2018, 122, 686-701. [CrossRef]

3. Arraiza, M.P.; González-Coloma, A.; Andres, M.F.; Berrocal-Lobo, M.; Domínguez-Núñez, J.A.; Da Costa, A.C., Jr.; Navarro-Rocha, J.; Calderón-Guerrero, C. Antifungal effect of essential oils. In Potential Essent. Oils; Hany El-Shemy, Ed.; IntechOpen: London, UK, 2018; pp. 145-148.

4. Kala, S.; Sogan, N.; Naik, S.N.; Patanjali, P.K.; Kumar, J. Biopesticides: Formulations and Delivery Techniques. In Natural Remedies for Pest, Disease and Weed Control; Egbuna, C., Sawicka, B., Eds.; Elsevier Inc.: Amsterdam, The Netherlands, 2020; pp. 209-220.

5. Lengai, G.M.W.; Muthomi, J.W.; Mbega, E.R. Phytochemical activity and role of botanical pesticides in pest management for sustainable agricultural crop production. Sci. Afr. 2020, 7, e00239. [CrossRef]

6. Campos, E.V.R.; Proença, P.L.F.; Oliveira, J.L.; Bakshi, M.; Abhilash, P.C.; Fraceto, L.F. Use of botanical insecticides for sustainable agriculture: Future perspectives. Ecol. Indic. 2019, 105, 483-495. [CrossRef]

7. Loi, M.; Paciolla, C.; Logrieco, A.F.; Mulè, G. Plant bioactive compounds in pre-and postharvest management for aflatoxins reduction. Front. Microbiol. 2020, 11, 243. [CrossRef] [PubMed]

8. Jamwal, K.; Bhattacharya, S.; Puri, S. Plant growth regulator mediated consequences of secondary metabolites in medicinal plants. J. Appl. Res. Med. Aromat. Plants 2018, 9, 26-38. [CrossRef]

9. Raveau, R.; Fontaine, J.; Lounès-Hadj Sahraoui, A. Essential oils as potential alternative biocontrol products against plant pathogens and weeds: A Review. Foods 2020, 9, 365. [CrossRef] [PubMed]

10. Arora, H.; Sharma, A.; Sharma, S.; Haron, F.F.; Gafur, A.; Sayyed, R.Z.; Datta, R. Pythium damping-off and root rot of capsicum annuum 1.: Impacts, diagnosis, and management. Microorganisms 2021, 9, 823. [CrossRef]

11. Blancard, D. Tomato Diseases: Identification, Biology and Control: A Colour Handbook; CRC Press: Boca Raton, FL, USA, 2019.

12. Kadoglidou, K.; Chatzopoulou, P.; Maloupa, E.; Kalaitzidis, A.; Ghoghoberidze, S.; Katsantonis, D. Mentha and oregano soil amendment induces enhancement of tomato tolerance against soilborne diseases, yield and quality. Agronomy 2020, 10, 406. [CrossRef]

13. Ozkaya, H.O.; Ergun, T. The effects of Allium tuncelianum extract on some important pathogens and total phenolic compounds in tomato and pepper. Pak. J. Bot 2017, 49, 2483-2490.

14. Singh, V.K.; Singh, A.K.; Kumar, A. Disease management of tomato through PGPB: Current trends and future perspective. 3 Biotech 2017, 7, 255. [CrossRef]

15. Borges, D.F.; Lopes, E.A.; Moraes, A.R.F.; Soares, M.S.; Visôtto, L.E.; Oliveira, C.R.; Valente, V.M.M. Formulation of botanicals for the control of plant-pathogens: A review. Crop Prot. 2018, 110, 135-140. [CrossRef]

16. Rongai, D.; Pulcini, P.; Pesce, B.; Milano, F. Antifungal activity of pomegranate peel extract against Fusarium wilt of tomato. Eur. J. Plant Pathol. 2017, 147, 229-238. [CrossRef]

17. De Rodriguez, D.J.; Gaytán-Sánchez, N.A.; Rodríguez-García, R.; Hernández-Castillo, F.D.; Díaz-Jiménez, L.; VillarrealQuintanilla, J.A.; Flores-López, M.L.; Carrillo-Lomelí, D.A.; Peña-Ramos, F.M. Antifungal activity of Juglans spp. and Carya spp. ethanol extracts against Fusarium oxysporum on tomato under greenhouse conditions. Ind. Crops Prod. 2019, 138, 111442. [CrossRef]

18. Hajji-Hedfi, L.; Larayedh, A.; Hammas, N.C.; Regaieg, H.; Horrigue-Raouani, N. Biological activities and chemical composition of Pistacia lentiscus in controlling Fusarium wilt and root-knot nematode disease complex on tomato. Eur. J. Plant Pathol. 2019, 155, 281-291. [CrossRef]

19. Hlokwe, M.T.P.; Kena, M.A.; Mamphiswana, N.D. Evaluating crude extracts of Monsonia burkeana and Moringa oleifera against Fusarium wilt of tomato. Acta Agric. Scand. Sect. B Soil Plant Sci. 2018, 68, 757-764. [CrossRef]

20. Mu'nisa, A.; Hiola, S.F.; Ali, A. The effectiveness of the formulation of Cocoa Pod Husk (Theobroma cacao L.) based botanical fungicides on Fusarium wilt disease on tomato plants. J. Phys. Conf. Ser. 2019, 1244, 012036. [CrossRef]

21. Ramírez, P.G.; Ramírez, D.G.; Mejía, E.Z.; Ocampo, S.A.; Díaz, C.N.; Rojas Martínez, R.I. Extracts of Stevia rebaudiana against Fusarium oxysporum associated with tomato cultivation. Sci. Hortic. (Amsterdam) 2020, 259, 108683. [CrossRef] 
22. Ben-Jabeur, M.; Ghabri, E.; Myriam, M.; Hamada, W. Thyme essential oil as a defense inducer of tomato against gray mold and Fusarium wilt. Plant Physiol. Biochem. 2015, 94, 35-40. [CrossRef]

23. Sharma, A.; Rajendran, S.; Srivastava, A.; Sharma, S.; Kundu, B. Antifungal activities of selected essential oils against Fusarium oxysporum f. sp. lycopersici 1322, with emphasis on Syzygium aromaticum essential oil. J. Biosci. Bioeng. 2017, 123, 308-313. [CrossRef]

24. Sharma, A.; Sharma, N.K.; Srivastava, A.; Kataria, A.; Dubey, S.; Sharma, S.; Kundu, B. Clove and lemongrass oil based non-ionic nanoemulsion for suppressing the growth of plant pathogenic Fusarium oxysporum f.sp. lycopersici. Ind. Crops Prod. 2018, 123, 353-362. [CrossRef]

25. Smaili, A.; Mazoir, N.; Rifai, L.A.; Koussa, T.; Makroum, K.; Kabil, E.M.; Benharref, A.; Faize, M. Triterpene derivatives from Euphorbia enhance resistance against Verticillium wilt of tomato. Phytochemistry 2017, 135, 169-180. [CrossRef] [PubMed]

26. Smaili, A.; Rifai, L.A.; Mazoir, N.; Koussa, T.; Faize, L.; Alburquerque, N.; Burgos, L.; Makroum, K.; Malika, B.; Benharref, A.; et al. Semisynthetic Triterpenes derived from Euphorbia officinarum as plant growth promoters and inducers of disease resistance. J. Plant Growth Regul. 2019, 38, 262-272. [CrossRef]

27. Mekam, P.N.; Martini, S.; Nguefack, J.; Tagliazucchi, D.; Stefani, E. Phenolic compounds profile of water and ethanol extracts of Euphorbia hirta L. leaves showing antioxidant and antifungal properties. S. Afr. J. Bot. 2019, 127, 319-332. [CrossRef]

28. La Torre, A.; Caradonia, F.; Matere, A.; Battaglia, V. Using plant essential oils to control Fusarium wilt in tomato plants. Eur. J. Plant Pathol. 2016, 144, 487-496. [CrossRef]

29. Helal, I.M. Control of damping-off disease in some plants using environmentally safe biocides. Pakistan J. Bot. 2017, 49, 361-370.

30. Kalleli, F.; Abid, G.; Ben Salem, I.; Boughalleb-M'Hamdi, N.; M'Hamdi, M. Essential oil from fennel seeds (Foeniculum vulgare) reduces Fusarium wilt of tomato (Solanum lycopersicon). Phytopathol. Mediterr. 2020, 59. [CrossRef]

31. Nefzi, A.; Abdallah, R.A.B.; Jabnoun-Khiareddine, H.; Ammar, N.; Medimagh-Saïdana, S.; Haouala, R.; Daami-Remadi, M. Management of Fusarium crown and root rot of tomato by Solanum linnaeanum L. extracts. Sci. Hortic. (Amsterdam) 2018, 238, 204-214. [CrossRef]

32. Nefzi, A.; Jabnoun-Khiareddine, H.; Aydi Ben Abdallah, R.; Ammar, N.; Medimagh-Saïdana, S.; Haouala, R.; Daami-Remadi, M. Suppressing Fusarium crown and root rot infections and enhancing the growth of tomato plants by Lycium arabicum Schweinf. Ex Boiss. extracts. S. Afr. J. Bot. 2017, 113, 288-299. [CrossRef]

33. Akladious, S.A.; Isaac, G.S.; Abu-Tahon, M.A. Induction and resistance against Fusarium wilt disease of tomato by using sweet basil (Ocimum basilicum L.) extract. Can. J. Plant Sci. 2015, 95, 689-701. [CrossRef]

34. Mekam, P.N.; Martini, S.; Nguefack, J.; Tagliazucchi, D.; Mangoumou, G.N.; Stefani, E. Activity of extracts from three tropical plants towards fungi pathogenic to tomato (Solanum lycopersicum). Phytopathol. Mediterr. 2019, 58, 573-586. [CrossRef]

35. Biswas, S.K.; Prakesh, H.G.; Palat, R.; Bahar, J. Induced synthesis of defense molecules in tomato (Solamum lycopercicum L.) against Fusarium wilt through plant extracts. Bangladesh J. Bot. 2019, 48, 169-175. [CrossRef]

36. Li, H.Y.; Zhou, X.G.; Wu, F.Z. Effects of root exudates from potato onion on Verticillium dahliae. Allelopath. J. 2018, 43, 217-222. [CrossRef]

37. Zhang, X.; Wang, H.; Zhu, W.; Li, W.; Wang, F. Transcriptome analysis reveals the effects of Chinese Chive (Allium tuberosum R.) extract on Fusarium oxysporum f. sp. radicis-lycopersici spore germination. Curr. Microbiol. 2020, 77, 855-864. [CrossRef] [PubMed]

38. More, Y.D.; Gade, R.M.; Shitole, A.V. Evaluation of antifungal activities of extracts of Aegle marmelos, Syzygium cumini and Pongamia pinnata against Pythium debaryanum. Indian J. Pharm. Sci. 2017, 79, 377-384. [CrossRef]

39. De Rodríguez, D.J.; Trejo-González, F.A.; Rodríguez-García, R.; Díaz-Jimenez, M.L.V.; Sáenz-Galindo, A.; Hernández-Castillo, F.D.; Villarreal-Quintanilla, J.A.; Peña-Ramos, F.M. Antifungal activity in vitro of Rhus muelleri against Fusarium oxysporum f. sp. lycopersici. Ind. Crops Prod. 2015, 75, 150-158. [CrossRef]

40. Chohan, S.; Perveen, R. Phytochemical analysis and antifungal efficacy of rhizome extracts of various plants against Fusarium wilt and root rot of tomato. Int. J. Agric. Biol. 2015, 17, 1193-1199. [CrossRef]

41. Muthomi, J.W.; Lengai, G.M.W.; Wagacha, M.J.; Narla, R.D. In vitro activity of plant extracts against some important plant pathogenic fungi of tomato. Aust. J. Crop Sci. 2017, 11, 683-689. [CrossRef]

42. Khurshid, S.; Javaid, A.; Shoaib, A.; Javed, S.; Qaisar, U. Antifungal activity of aerial parts of Cenchrus pennisetiformis against Fusarium oxysporum f. sp. lycopersici. Planta Daninha 2018, 36, 1-10. [CrossRef]

43. Nugroho, C.; Mirnia, E.; Cumagun, C.J.R. Antifungal activities of sweet basil (Ocimum basilicum L.) Aqueous Extract Against against Sclerotium rolfsii, causal agent of damping-off on tomato seedling. Agrivita J. Agric. Sci. 2019, 41, 149-157. [CrossRef]

44. Wang, Y.; Sun, Y.; Zhang, Y.; Zhang, X.; Feng, J. Antifungal activity and biochemical response of cuminic acid against Phytophthora capsici Leonian. Molecules 2016, 21, 756. [CrossRef]

45. Švecová, E.; Colla, G.; Crinò, P. Antifungal activity of Boerhavia diffusa L. extract against Phytophthora spp. in tomato and pepper Eur. J. Plant Pathol. 2017, 148, 27-34. [CrossRef]

46. Amini, J.; Farhang, V.; Javadi, T.; Nazemi, J. Antifungal effect of plant essential oils on controlling Phytophthora species. Plant Pathol. J. 2016, 32, 16-24. [CrossRef] [PubMed]

47. Pandey, M.; Ahmad, S.; Khan, K.Z. Efficacy of selected plant extracts and biocontrol agents against damping-off (Pythium aphanidermatum) of chilli. Res. Environ. Life Sci. 2017, 10, 87-90. 
48. Rajput, N.A.; Atiq, M.; Javed, N.; Ye, Y.H.; Zhao, Z.; Syed, R.N.; Lodhi, A.M.; Khan, B.; Iqbal, O.; Dou, D. Antimicrobial effect of Chinese medicinal plant crude extracts against Rhizoctonia solani and Pythium aphanidermatum. Fresenius Environ. Bull. 2018, 27, 3941-3949.

49. Liu, X.; Ouyang, C.; Wang, Q.; Li, Y.; Yan, D.; Yang, D.; Fang, W.; Cao, A.; Guo, M. Effects of oil extracts of Eupatorium adenophorum on Phytophthora capsici and other plant pathogenic fungi in vitro. Pestic. Biochem. Physiol. 2017, 140, 90-96. [CrossRef]

50. Abou-Zeid, N.; Mahmoud, N.; Saleh, R. Effect of some biotic and abiotic applications on control of Fusarium wilt of Pepper plants. Egypt. J. Phytopathol. 2016, 44, 103-118. [CrossRef]

51. Chen, F.J.; Long, X.H.; Li, E.Z. Evaluation of antifungal phenolics from Helianthus tuberosus L. leaves against Phytophthora capsici leonian by chemometric analysis. Molecules 2019, 24, 4300. [CrossRef]

52. Mongiano, G.; Zampieri, E.; Morcia, C.; Titone, P.; Volante, A.; Terzi, V.; Tamborini, L.; Valé, G.; Monaco, S. Application of plant-derived bioactive compounds as seed treatments to manage the rice pathogen Fusarium fujikuroi. Crop Prot. 2021, 148, 105739. [CrossRef]

53. Saghrouchni, H.; El Barnossi, A.; Salamatullah, A.M.; Bourhia, M.; Alzahrani, A.; Alkaltham, M.S.; Alyahya, H.K.; Tahiri, N.E.H.; Imtara, H.; Var, I. Carvacrol: A promising environmentally friendly agent to fight seeds damping-off diseases induced by fungal species. Agronomy 2021, 11, 985. [CrossRef]

54. Zhao, Y.; Wang, Q.; Wu, X.; Jiang, M.; Jin, H.; Tao, K.; Hou, T. Unraveling the polypharmacology of a natural antifungal product, eugenol, against Rhizoctonia solani. Pest Manag. Sci. 2021, 77, 3469-3483. [CrossRef]

55. Baysal-Gurel, F.; Liyanapathiranage, P.; Addesso, K.M. Effect of Brassica crop-based biofumigation on soilborne disease suppression in woody ornamentals. Can. J. Plant Pathol. 2020, 42, 94-106. [CrossRef]

56. Pane, C.; Villecco, D.; Zaccardelli, M. Combined use of Brassica carinata seed meal, thyme oil and a Bacillus amyloliquefaciens strain for controlling three soil-borne fungal plant diseases. J. Plant Pathol. 2017, 99, 77-84. [CrossRef]

57. Ma, Y.; Gentry, T.; Hu, P.; Pierson, E.; Gu, M.; Yin, S. Impact of brassicaceous seed meals on the composition of the soil fungal community and the incidence of Fusarium wilt on chili pepper. Appl. Soil Ecol. 2015, 90, 41-48. [CrossRef]

58. Al-Hammouri, A.A.; Al-Kofahi, S.D.; Ibbini, J.H.; Abusmier, S.A.; Sanogo, S. Effect of biofumigation by Calligonum polygonoides, dry olive leaves, and ash of olive leaves on chilli pepper growth and recovery of Rhizoctonia solani. Acta Agric. Slov. 2018, 111, 41-49. [CrossRef]

59. Gurjar, M.S.; Ali, S.; Akhtar, M.; Singh, K.S. Efficacy of plant extracts in plant disease management. Agric. Sci. $2012,3,425-433$. [CrossRef]

60. Balouiri, M.; Sadiki, M.; Ibnsouda, S.K. Methods for in vitro evaluating antimicrobial activity: A review. J. Pharm. Anal. 2016, 6, 71-79. [CrossRef]

61. Favre-Godal, Q.; Queiroz, E.F.; Wolfender, J.L. Latest developments in assessing antifungal activity using TLC-bioautography: A review. J. AOAC Int. 2013, 96, 1175-1188. [CrossRef]

62. Cairns, T.C.; Studholme, D.J.; Talbot, N.J.; Haynes, K. New and improved techniques for the study of pathogenic fungi. Trends Microbiol. 2016, 24, 35-50. [CrossRef]

63. Shanmugam, G.; Jeon, J. Computer-aided drug discovery in plant pathology. Plant Pathol. J. 2017, 33, 529-542. [CrossRef]

64. Kar, S.; Roy, K.; Leszczynski, J. On Applications of QSARs in Food and Agricultural Sciences: History and Critical Review of Recent Developments. In Advances in QSAR Modeling: Applications in Pharmaceutical, Chemical, Food, Agricultural and Environmental Sciences; Roy, K., Ed.; Springer International Publishing: Cham, Switzerland, 2017; pp. 203-302.

65. Amoroso, V.B.; Mendez, R.A.; Junio, H.A.; Molino, R.J.E.J.; Pescadero, I.R.; Villalobos, A.P. Characterization of a natural fungicide from an indigenous plant Tasmannia piperita (Hook. f.) Miers Extract: Stability, Metabolomics, and In silico Studies. Philipp. J. Sci. 2021, 150, 355-370.

66. Sidhu, K.S.; Bhangu, S.K.; Pathak, R.K.; Yadav, I.S.; Chhuneja, P. Identification of natural lead compounds for leaf rust of Wheat: A molecular docking and simulation study. J. Proteins Proteomics 2020, 11, 283-295. [CrossRef]

67. Pontes, J.G.D.M.; Fernandes, L.S.; Dos Santos, R.V.; Tasic, L.; Fill, T.P. Virulence factors in the phytopathogen-host interactions: An overview. J. Agric. Food Chem. 2020, 68, 7555-7570. [CrossRef] [PubMed]

68. Marquez, N.; Giachero, M.L.; Declerck, S.; Ducasse, D.A. Macrophomina phaseolina: General characteristics of pathogenicity and methods of control. Front. Plant Sci. 2021, 12, 634397. [CrossRef] [PubMed]

69. Sorokina, M.; Steinbeck, C. Review on natural products databases: Where to find data in 2020. J. Cheminform. 2020, 12, 20. [CrossRef] [PubMed]

70. Valdés-Jiménez, A.; Peña-Varas, C.; Borrego-Muñoz, P.; Arrue, L.; Alegría-Arcos, M.; Nour-Eldin, H.; Dreyer, I.; Nuñez-Vivanco, G.; Ramírez, D. Psc-db: A structured and searchable 3d-database for plant secondary compounds. Molecules 2021, $26,1124$. [CrossRef] [PubMed]

71. Silva, J.K.R.D.; Silva, J.R.A.; Nascimento, S.B.; Luz, S.F.M.D.; Meireles, E.N.; Alves, C.N.; Ramos, A.R.; Maia, J.G.S. Antifungal activity and computational study of constituents from Piper divaricatum essential oil against Fusarium infection in black pepper. Molecules 2014, 19, 17926-17942. [CrossRef]

72. Campos, V.A.; Perina, F.J.; Alves, E.; Sartorelli, J.; Moura, A.M.; Oliveira, D.F. Anadenanthera colubrina (Vell.) Brenan produces steroidal substances that are active against Alternaria alternata (Fr.) Keissler and that may bind to oxysterol-binding proteins. Pest Manag. Sci. 2014, 70, 1815-1822. [CrossRef] 
73. Priyadharsini, K.; Saravanakumar, S.; Bharathi, N.; Ramalingam, J. Exploring the competence of phytochemical compounds to combat anthracnose disease of cucumber-an in silico approach. Int. J. Pharm. Pharm. Sci. 2014, 6, 255-258.

74. Pani, G.; Dessì, A.; Dallocchio, R.; Scherm, B.; Azara, E.; Delogu, G.; Migheli, Q.M. Natural phenolic inhibitors of trichothecene biosynthesis by the wheat fungal pathogen Fusarium culmorum: A computational insight into the structure-activity relationship. PLoS ONE 2016, 11, e0157316. [CrossRef]

75. Kessler, A.; Kalske, A. Plant secondary metabolite diversity and species interactions. Annu. Rev. Ecol. Evol. Syst. 2018, 49, 115-138. [CrossRef]

76. Isman, M.B. Botanical Insecticides in the Twenty-First Century-Fulfilling Their Promise ? Annu. Rev. 2020, 233-251. [CrossRef] [PubMed]

77. Ivase, T.J.-P.; Nyakuma, B.B.; Ogenyi, B.U.; Balogun, A.D.; Hassan, M.N. Current status, challenges and prospects of biopesticide utilization in Nigeria. Acta Univ. Sapientiae Agric. Environ. 2017, 9, 95-106. [CrossRef]

78. Keswani, C.; Dilnashin, H.; Birla, H.; Singh, S.P. Regulatory barriers to Agricultural Research commercialization: A case study of biopesticides in India. Rhizosphere 2019, 11, 100155. [CrossRef]

79. Chandler, D.; Bailey, A.S.; Tatchell, G.M.; Davidson, G.; Greaves, J.; Grant, W.P. The development, regulation and use of biopesticides for integrated pest management. Philos. Trans. R. Soc. B Biol. Sci. 2011, 366, 1987-1998. [CrossRef]

80. Meneguzzo, F.; Zabini, F. Sustainable Crop Protection and Farming. In Agri-Food and Forestry Sectors for Sustainable Development; Sustainable Development Goals Series; Meneguzzo, F., Zabini, F., Eds.; Springer International Publishing: Cham, Switzerland, 2021; pp. 55-65.

81. Lykogianni, M.; Bempelou, E.; Karamaouna, F.; Aliferis, K.A. Do pesticides promote or hinder sustainability in agriculture? The challenge of sustainable use of pesticides in modern agriculture. Sci. Total Environ. 2021, 795, 148625. [CrossRef]

82. OECD. Joint Meeting of the Chemicals Committee and the Working Party on Chemicals, Pesticides and Biotechnology Guidance Document on Botanical Active Substances Used in Plant Protection Products; Series on Pesticides No. 90 JT03412077; OECD Environment, Health and Safety Publications: Paris, France, 2017.

83. Mishra, J.; Dutta, V.; Arora, N.K. Biopesticides in India: Technology and sustainability linkages. 3 Biotech 2020, 10, 210. [CrossRef]

84. Damalas, C.A.; Koutroubas, S.D. Current status and recent developments in biopesticide use. Agriculture 2018, 8, 13. [CrossRef] 